\title{
Systemic Mastocytosis with an Associated Hematological Neoplasm
}

National Cancer Institute

\section{Source}

National Cancer Institute. Systemic Mastocytosis with an Associated Hematological

Neoplasm. NCl Thesaurus. Code C9284.

A disorder characterized by systemic infiltration of internal organs by aggregates of neoplastic mast cells and the presence of a clonal non-mast cell hematologic neoplasm (e.g., myelodysplastic syndrome, chronic myeloproliferative disorder, acute myeloid leukemia, and lymphoma). 